\title{
AS EXPERIÊNCIAS DOS CUIDADOS DE SAÚDE DE PESSOAS TRANSEXUAIS EM PORTUGAL: PERSPETIVAS DE PROFIS- SIONAIS DE SAÚDE E UTENTES ${ }^{1}$
}

\author{
Nuno Pinto ${ }^{2}$ \\ Carla Moleiro ${ }^{3}$
}

Resumo: O presente artigo pretende contribuir para dois objetivos, um de caráter teórico e outro de natureza empírica, no contexto português em que recentemente o papel dos/as profissionais de saúde se tornou mais relevante na avaliação clínica, apoio e mudança de nome/sexo legal de pessoas transexuais. Deste modo, o primeiro objetivo consiste em rever a literatura e os "standards of care" internacionais no que diz respeito aos cuidados de saúde com pessoas transexuais. O segundo objetivo remete para a apresentação de um estudo empírico qualitativo, que explora as perspetivas de clínicos/as $(\mathrm{n}=6)$ e de pessoas transexuais $(\mathrm{n}=7)$ sobre as suas experiências em serviços de saúde em Portugal. Os resultados são demonstrativos de certas competências destas equipas clínicas, mas também alertam para a existência de práticas contrárias às recomendações internacionais. Estes resultados são discutidos à luz da literatura respeitante à transexualidade mas também das abordagens relativas à sensibilidade clínica para a diversidade individual e cultural.

Palavras-chave: transexuais; cuidados clínicos; competências para a diversidade individual e cultural; estudo qualitativo.

Experiences of Health Care by Transexual People in Portugal: The perspectives of health professionals and health care users (Abstract): The present paper aims to contribute to two objetives, one of a theoretical nature and one of empirical

${ }^{1}$ Agradecimentos: Este estudo foi realizado ao abrigo de uma colaboração com o Projeto TRANSFormation - da responsabilidade da Associação ILGA Portugal e financiado pela ILGA Europe.

${ }^{2}$ Estudante de Doutoramento - Instituto Universitário de Lisboa - ISCTE-IUL/CIS. A correspondência relativa a este artigo deve ser endereçada a Nuno Pinto, CIS-IUL/Centro de Investigação e Intervenção Social - Instituto Universitário de Lisboa, Edifício ISCTE, Avenida das Forças Armadas, 1649-026 Lisboa; Email: nuno.pinto@iscte.pt

3 Professora Auxiliar - Instituto Universitário de Lisboa - ISCTE-IUL/CIS 
nature, in the Portuguese context where recently the role of clinicians has become more relevant in assessment, support, sex reassignment and legal change of sex for transsexual persons. Hence, the first goal consists in a review of the literature and international standards of care for the treatment of transexual persons. The second goal involves the presentation of an empirical qualitative study, exploring the perspetive of clinicians $(n=6)$ and transsexual persons $(n=7)$ regarding their experiences of health care in Portugal. The results show certain competences and skills of these clinical teams, but also reveal the existence of practices contrary to international recommendations. These results are discussed in light of the literature concerning transsexuality but also of the approaches to the clinical sensitivity to individual and cultural diversity.

Keywords: transsexuals; clinical care; individual and cultural diversity competence; qualitative study.

\section{Introdução e revisão da literatura}

Em Portugal, a recente aprovação de uma Lei de Identidade de Género, que vem permitir a mudança de sexo legal e de nome próprio no registo civil, trouxe alguma atenção pública à temática da transexualidade. Tal aconteceu em contraponto à sistemática invisibilidade que historicamente tem marcado as trajetórias de vida das pessoas transexuais. Este vazio social (Saleiro, 2009) tem o seu paralelo a nível da investigação, especialmente no que respeita às ciências sociais. Em contexto português, os estudos sobre orientação sexual e identidade de género encontram-se ainda numa fase embrionária (Vale de Almeida, 2010). Especificamente, na temática transexual/transgénero é evidente a ausência de trabalhos realizados, com exceção para alguns autores das ciências médicas (Saleiro, 2009). Consequentemente, em Portugal, os dados disponíveis sobre o acompanhamento clínico a pessoas transexuais são marcadamente incipientes, e a produção de recomendações de boas práticas é inexistente. Procurando contrariar esta lacuna, o presente estudo aspira promover a investigação relativa à situação dos cuidados clínicos às pessoas transexuais em Portugal, e contribuir para o conhecimento científico e crítico acerca dos mesmos. São dois os objetivos a que nos propomos: um de caráter teórico, que remete para a revisão da literatura e das diretrizes internacionais sobre o acompanhamento clínico a pessoas transexuais; e outro de natureza empírica, que se concretiza na apresentação de um estudo qualitativo que explora as perspetivas de clínicos/as e de pessoas transexuais sobre as suas experiências em serviços de saúde em Portugal.

Entende-se por identidade de género o reconhecimento pessoal, básico e profundo, em geral, enquanto mulher ou homem (American Psychological 
Association [APA], 2008). As pessoas transexuais são aquelas cuja identidade de género não é congruente com o sexo que lhes foi atribuído ao nascimento. Em geral, as pessoas transexuais vivem socialmente de modo concordante com a sua identidade de género, independentemente das intervenções médicas a que se tenham submetido ou do desejo de as fazer no futuro (APA, 2008). A noção de disforia de género refere-se ao desconforto ou sofrimento causado pela discrepância entre a identidade de género e o sexo atribuído à nascença (Knudson, Cuypere \& Bockting, 2010). As pessoas transexuais podem, ou não, experienciar disforia de género em determinados momentos da sua trajetória de vida (WPATH, 2011), e desejar submeter-se, ou não, a tratamentos cirúrgicos e/ou hormonais com vista ao seu tratamento (Lev, 2004). Há pessoas transexuais que, num determinado momento da vida, sentem a disforia de género de modo tão intenso que esse mal-estar se traduz em dificuldade marcada de funcionamento social, ocupacional, ou noutras áreas importantes do seu desenvolvimento, cumprindo os critérios para o estabelecimento de um diagnóstico no domínio da saúde mental - tal como o diagnóstico de Perturbação de Identidade de Género, patente no DSM-IV (American Psychiatric Association, 2004). O conceito de transgénero é atualmente usado para referir o comportamento, a aparência ou a identidade de pessoas que perturbam, transcendem ou não se conformam com as normas culturais definidas para pessoas do seu sexo biológico (APA, 2008). Este termo é usado de modo inclusivo, abarcando as próprias pessoas transexuais, mas também pessoas intersexuais, travestis, drag queens, entre outras (Hines, 2007a).

\section{Situação social e saúde mental}

Os dados sobre a prevalência das pessoas transexuais em Portugal são inexistentes. A informação disponível sustenta-se nos relatos informais de profissionais de saúde (Saleiro, 2009) e nos dados dos respetivos serviços (Albuquerque, 2006), relativos aos processos clínicos que acompanham. Não obstante, os relatos são, em geral, demonstrativos da maior utilização dos serviços por homens transexuais ${ }^{4}$, comparativamente às mulheres transexuais. Este rácio é contrário à tendência encontrada em diversos estudos realizados noutros países, que apontam para uma maior prevalência de mulheres transexuais (Bakker, van Kesteren, Gooren \& Bezemer, 1993; Garrels, Kockott, Michael et al., 2000; Olsson \& Moller, 2003).

\footnotetext{
${ }^{4}$ Faremos uso da linguagem de modo concordante com a identidade de género. Homens transexuais serão, assim, pessoas cujo sexo atribuído à nascença foi feminino e cuja identidade de género é masculina. Por sua vez, mulheres transexuais serão as pessoas cujo sexo atribuído à nascença foi masculino e cuja identidade de género é feminina.
} 
A população transexual é heterogénea e muito diversa entre si. Os/as transexuais podem ser adolescentes, adultos/as ou idosos/as; constituir diferentes configurações familiares, ser hetero, homo ou bissexuais, podendo ou não - ter filhos/as; pertencem a qualquer nível socioeconómico, e desempenham as mais variadas atividades laborais; inserem-se, ainda, em diferentes grupos étnicos e religiosos (Raj, 2002). Um recente estudo nacional (Costa, Pereira, Oliveira \& Nogueira, 2010) encontrou que as pessoas percebidas como as mais discriminadas, por entre uma listagem de mais de 20 grupos sociais minoritários, são as/os transexuais. De facto, as pessoas transexuais são frequentemente vítimas de diferentes formas de discriminação, desde a alienação económica (Clements, Wilkinson, Kitano \& Marx, 1999; Lombardi, Wilchins, Priesing \& Malouf, 2001) até à reiterada vitimização decorrente do abuso físico e psicológico (Lombardi et al., 2001; Nuttbrock, Hwahng, Bockting et al., 2010).

Em paralelo, diferentes estudos encontram resultados consensuais no que respeita a níveis inferiores de saúde mental/bem-estar psicológico e de qualidade de vida, comparativamente com a população média - seja como corolário da referida discriminação e alienação social (APA, 2008; Nuttbrock et al., 2010; Sánchez \& Vilain, 2009), seja como produto dos eventuais conflitos internos relativos ao género decorrentes da própria condição de transexual (Dean, Meyer, Robinson et al., 2000; Newfield, Hart, Dibble \& Kohler, 2006). Deste modo, as pessoas transexuais têm sido assinaladas como em maior risco para desenvolver, por exemplo, perturbações de ansiedade (Hepp, Kramer, Schnyder, Miller, \& Delsignore, 2005), depressão (Nuttbrock et al., 2010), fobia social e perturbações de ajustamento (Gómes-Gil, Trilla, Salamero et al., 2009) abuso de substâncias (Lawrence, 2008) ou perturbações do comportamento alimentar (Vocks, Stahn, Loenser \& Tegenbauer, 2009). Em simultâneo, os dados relativos à ideação e tentativa de suicídio nesta população são salientes: Maguen e Shipherd (2010) encontraram valores de tentativa de suicídio na ordem dos $40 \%$ em homens transexuais e $20 \%$ em mulheres transexuais; Nuttbrock e colegas (2010), num recente estudo com cerca de 500 mulheres transexuais, indicam que cerca de $30 \%$ já se tentou suicidar, cerca de $35 \%$ já planeou fazê-lo e perto de metade das participantes já pensou nisso.

O bem-estar e a saúde mental de jovens transgénero, nomeadamente das/os transexuais, só recentemente começou a merecer destaque na investigação psicológica (Dean et al., 2000). A adolescência tem sido apontada como uma fase de maior risco no que respeita à saúde mental de pessoas transgénero. Tal reflete, por um lado, a emergência da puberdade e o desenvolvimento das características sexuais secundárias - muitas vezes acompanhada por sentimentos de isolamento e de repulsa pelo próprio corpo (Korell \& Lorah, 2007); por outro, os abusos psicológicos e físicos que podem 
caracterizar as relações interpessoais nesta fase da vida, que surgem associados aos comportamentos e às expressões de género percebidas por outros/as como não normativas (Nuttbrock et al., 2010). Deste modo, os dados indicativos do risco para a saúde mental e o bem-estar psicológico de pessoas transexuais acentuam-se se nos focarmos em adolescentes ou jovens adultos. Por exemplo, Israel e Tarver (1997) indicam que entre $50 \%$ a $88 \%$ de jovens transgénero já consideraram ou tentaram suicidar-se.

Não raramente, a discriminação e os abusos psicológicos e físicos de que as pessoas transexuais são alvo ocorrem no contexto de relações interpessoais significativas, como as relações familiares ou laborais (Lombardi et al., 2001; Nuttbrock et al., 2010; Kenagy, 2005). Um crescente, mas ainda incipiente, número de investigações tem abordado as idiossincrasias das relações familiares e/ou amorosas, bem como das experiências laborais desta população (por exemplo, Ettner, 2007; Budge, Tebbe \& Howard, 2010). Não obstante, a família é um dos sistemas de suporte ao qual as pessoas transexuais recorrem (Korell \& Lorah, 2007), de modo condicionado pelas reconfigurações e dinâmicas familiares despoletadas pelo assumir da transexualidade (Emerson \& Rosenfeld, 1996). Também a própria comunidade transexual, nomeadamente as associações e grupos de apoio, tem sido apontada como um recurso do qual esta população pode beneficiar (Hinnes, 2007b; Korell \& Lorah, 2007; Lev, 2007). A participação em grupos de apoio, mais ou menos formais, está relacionada com diferentes dimensões (Hinnes, 2007b): a possibilidade de ser honesto/a no que respeita à sua identidade de género e de a assumir perante outros/as; a procura de apoio, não apenas emocional mas também informativo/educativo; ou ainda, a possibilidade de não só receber apoio, mas também o ministrar. Os grupos de apoio, que frequentemente partem de (ou se limitam a) comunidades online (Lev, 2007), são muitas vezes identificados como vitais em colmatar a insuficiência de informação e de recursos educativos prestados pela comunidade médica (Hinnes, 2007b). Os/as transexuais dependem extensamente dos/as profissionais de saúde, nomeadamente os/as que trabalham na área da saúde mental, não apenas para atos médicos, mas também para acederem a informação credível relativamente a questões no domínio da transexualidade (Korell \& Lorah, 2007).

\section{Cuidados clínicos}

Nem todos/as os/as transexuais necessitam e/ou desejam apoio médico e psicológico (Raj, 2002). Não obstante, a comunidade médica é, em paralelo com a família e a própria comunidade transexual, um sistema de apoio basilar para as pessoas transexuais (Korell \& Lorah, 2007). Para a maioria das pessoas com dúvidas acerca da sua identidade de género, o primeiro passo parece ser a procura de um profissional de saúde - nomeadamente o seu médico de família (Hinnes, 2007b). 
Tal como exposto previamente, a condição de transexual coloca as pessoas em maior risco no que respeita à sua saúde mental. Os/as profissionais de saúde mental podem desempenhar um papel importante na promoção do bem-estar psicológico e da qualidade de vida desta população (Newfield, 2006). A investigação realça os efeitos positivos decorrentes das experiências de psicoterapia. Num estudo efetuado por Rachlin (2002), a maioria dos/as participantes relatou mudanças de vida positivas associadas a práticas psicoterapêuticas - mesmo quando sentiram que o/a terapeuta não tinha formação adequada em temáticas transgénero/transexuais ou, ainda, em situações nas quais não recomendariam o/a terapeuta a um/a amigo/a. É importante realçar que as pessoas transexuais procuram profissionais de saúde mental por variadas razões, não necessariamente relativas a questões relacionadas com a sua identidade de género (APA, 2008; Rachlin, 2002; Raj, 2002). De qualquer forma, as experiências de psicoterapia sentidas como mais positivas e apoiantes estão associadas à aceitação e ao respeito pela identidade de género, à flexibilidade na abordagem terapêutica e à proximidade com a comunidade transexual (Rachlin, 2002). Em paralelo, clientes de psicoterapia transexuais tendem a percecionar as dimensões "empatia" e "suporte" como essenciais no desenvolvimento de relações terapêuticas duradouras e produtivas (Bess \& Stabb, 2009).

Apesar do papel que os/as profissionais de saúde mental podem ter na promoção do bem-estar e qualidade de vida de pessoas transexuais, esta classe profissional tem maioritariamente intervindo em áreas relacionadas com o diagnóstico e a regulamentação do acesso aos atos médicos (Johnson, 2007). De facto, um dos papeis centrais que os/as profissionais de saúde mental desempenham junto de pessoas transexuais é o de gatekeepers $^{5}$, determinando quais os/as clientes adequados/as para acederem ao tratamento hormonal e às cirurgias de reatribuição sexual (May, 2002; Lev, 2007; Bess \& Stabb, 2009). Esta prática é regulada internacionalmente pelas recomendações da World Professional Association for Transgender Health (WPATH), exploradas em maior detalhe no ponto seguinte. Os resultados da investigação nesta área revelam que a função de gatekeeper despoleta uma dinâmica de poder entre cliente e terapeuta, colocando desafios específicos a ambas as partes da díade terapêutica e podendo afetar de modo decisivo a construção de uma relação de confiança e produtiva (Bess \& Stabb, 2009; Bockting, Robinso, Benner \& Scheltema, 2004; Raj, 2002). Alguns/algumas clientes adaptam inclusivamente as suas narrativas e histórias de vida, procurando ir de encontro ao que entendem ser as classificações diagnósticas rígi-

\footnotetext{
${ }^{5}$ Decidimos pela não tradução do termo original em inglês, uma vez que não encontrámos nenhum termo que em português exemplifique o significado atribuído a gatekeeper no contexto que aqui é usado. Uma possibilidade seria "zelador/a", ou ainda "porteiro/a".
} 
das e as perceções dos/as profissionais de saúde acerca do que é o género, as suas formas de expressão e, em particular, a transexualidade (Johnson, 2007; Lev, 2007; May, 2002). Neste sentido, tem sido sugerido que a avaliação psicológica seja distinguida da psicoterapia (quando necessária), e que um processo não seja substitutivo do outro (Rachlin, 2002). Aliás, clientes que procuram profissionais de saúde mental para conseguir aceder aos tratamentos hormonais e/ou cirúrgicos tendem a ter um número bastante inferior de sessões, do que aqueles/as que procuram os serviços por outras razões, como o desenvolvimento pessoal, apoio para situações específicas ou mesmo a exploração de questões relacionadas com o género (Bockting et al., 2004).

Além dos desafios específicos colocados pela função de gatekeeper, outros entraves têm sido encontrados no que respeita ao acesso das pessoas transexuais a cuidados de saúde mental apropriados, nomeadamente a dificuldade em encontrar clínicos/as com conhecimentos e competências específicas às questões transgénero e transexual (Sanchez, Sanchez \& Danoff, 2009). Dada a especificidade destas temáticas, tem sido sugerido que mesmo os/as terapeutas devidamente preparados/as para o trabalho clínico com outras minorias sexuais (como as pessoas lésbicas, gays e bissexuais) não estão necessariamente informados/as e aptos/as para trabalharem com clientes transexuais (Israel, 2005). A título de exemplo, muitos/as dos/as participantes do estudo de Korell e Lorah (2007) referiram ter sido os/as primeiros/as clientes transgénero/transexuais dos/as seus/suas terapeutas, e ter tido necessidade de os/as informar e educar em relação a estas temáticas - desencadeando sentimentos de frustração e ansiedade, tendo em conta que estes/as profissionais detêm o poder de autorizar (ou não) o acesso a tratamentos médicos. Em paralelo, mesmo os/as profissionais de saúde com conhecimentos no domínio da transexualidade não são necessariamente livres de estereótipos e preconceitos no que respeita à diversidade de expressões de género (Sanchez et al., 2009). De um modo geral, os discursos e as práticas médicas tendem a ser intolerantes à ambiguidade de género (May, 2002) e assentam em pressupostos heterossexistas (Johnson, 2007). Tal tem-se refletido no desenrolar de formas específicas de transfobia clínica, tais como a tendência para sobrepatologizar clientes transgénero/transexuais ou a perpetuação de mitos acerca das pessoas transgénero/transexuais, impostos pelas expectativas demonstradas pelos/as terapeutas, entre outras (Raj, 2002). As práticas psicoterapêuticas menos sensíveis à diversidade de expressões de género decorrem, por um lado, da falta de treino e formação específica para o trabalho com clientes transexuais e, por outro, do facto de os/as terapeutas serem um produto da sua própria socialização, estando provavelmente menos confortáveis no trabalho com clientes minoritários (Korell \& Lorah, 2007). 


\section{Standars of Care}

A WPATH é uma associação internacional constituída por profissionais de diferentes áreas, que tem como missão promover cuidados de saúde adequados e baseados em evidências científicas, formação, investigação, políticas públicas e respeito pelas pessoas transexuais. Tem publicado recomendações relativas ao acompanhamento clínico de pessoas transexuais, os chamados Standards of Care (SOC), que incidem em particular sobre os preceitos subjacentes às funções de gatekeeping. Tem sido sugerido que estas recomendações sejam abarcadas de modo crítico e complementadas por outras orientações (Raj, 2002; Lev, 2007). Não obstante, trata-se de um documento fundamental, de conhecimento essencial para qualquer profissional de saúde que trabalhe com pessoas transexuais. Realçamos aqui algumas das recomendações expressas na última versão (WPATH, 2011).

Estes standars clínicos explicitam que o objetivo da intervenção com as pessoas cuja identidade de género não é congruente com o sexo atribuído à nascença deve ser o de contribuir para o conforto pessoal e duradouro com o género identificado, de forma a maximizar o seu bem-estar global e a realização pessoal. Realçam que o tratamento é sempre individualizado: aquilo que ajuda uma pessoa a aliviar a disforia de género pode ser muito diferente daquilo que ajuda outras pessoas. Este processo pode, ou não, envolver mudanças nas expressões de género ou alterações corporais. As opções médicas incluem, por exemplo, a masculinização/feminização do corpo através de terapia hormonal e/ou cirúrgica - que podem efetivamente aliviar a disforia de género e que são clinicamente necessárias para muitas pessoas transexuais. Não obstante, os SOC são claros na defesa da diversidade de identidades e expressões de género, realçando que o recurso a hormonas e cirurgias são apenas duas opções por entre os vários recursos que podem ajudar as pessoas a sentirem-se confortáveis com a sua identidade de género.

Estas diretrizes internacionais indicam que qualquer profissional de saúde que acompanhe pessoas transexuais deve respeitar os seguintes princípios fundamentais: (1) revelar respeito pelos/as pacientes, independentemente da sua identidade e expressões de género (isto é, não patologizar diferentes expressões e identidades de género); (2) prestar cuidados de saúde (ou encaminhar para colegas mais bem preparados/as) orientados para a diminuição do mal-estar provocado pela disforia do género, de modo concordante com a identidade de género de cada um(a); (3) manter-se informado/a acerca das necessidades de saúde das pessoas transexuais, nomeadamente dos benefícios e dos riscos das diferentes opções de tratamento; (4) fazer corresponder o tratamento às necessidades e aos desejos específicos de cada paciente nomeadamente no que respeita às suas expressões de género; (5) facilitar o acesso a cuidados de saúde adequados; (6) obter o consentimento informado 
de cada paciente antes de iniciar qualquer tratamento; (7) prestar cuidados de saúde continuados; e (8) estar preparado/a para prestar apoio aos seus e às suas pacientes através da intervenção junto das famílias e comunidades.

Os SOC são claros ao afirmar que nenhum diagnóstico relacionado com a disforia de género pode ser motivo de estigmatização nem de privação de direitos, e que o diagnóstico é a descrição de um problema que a pessoa atravessa num determinado momento, não uma descrição da sua identidade. Em concreto, no que toca à função de gatekeeping, os SOC atribuem aos/às profissionais de saúde mental as funções relacionadas com a avaliação clínica e com a recomendação para a realização de tratamentos hormonais e/ou cirúrgicos. Para o início da terapia hormonal e/ou de cirurgias mamárias é exigida a recomendação de um(a) profissional de saúde mental (por exemplo, através de uma carta ou um relatório clínico), sendo que para as cirurgias genitais são exigidas recomendações de dois/duas profissionais de saúde mental ${ }^{6}$.

\section{Abordagens Trans-Positivas}

Em paralelo, têm surgido recomendações de diferentes autores/as orientadas para a promoção de cuidados clínicos que sejam sensíveis à diversidade de expressões de género. Raj (2002) propõe um contínuo entre duas posições opostas: os modelos de proveniência psiquiátrica, patologizantes, dirigidos para e pelos/as profissionais de saúde; e os modelos des-medicalizados, assentes em exclusivo na autodeterminação, dirigidos para e pelos/as consumidores/as. Entre estas posições extremas, assinaladas pelo autor como antiéticas, encontrar-se-ão as abordagens colaborativas e dirigidas para os/as clientes. Raj (2002) avança com uma série de recomendações para os/as profissionais de saúde que ambicionam prestar serviços e cuidados que sejam trans-positivos, isto é, sensíveis à diversidade de expressões de género. Realçamos apenas algumas destas recomendações: os/as profissionais devem procurar treino e formação especializada (e que ultrapasse a vertente do conhecimento, incidindo igualmente na dimensão das atitudes);

${ }^{6}$ Os SOC (WPATH, 2011) apontam vários critérios de elegibilidade/preparação - que devem constar nos relatórios clínicos - para os diferentes tratamentos médicos. Para as terapias hormonais e/ou cirurgias ao peito são indicados os seguintes: (1) disforia de género persistente e bem documentada; (2) capacidade de tomar decisões informadas e de consentir o tratamento; (3) ser maior de idade (com exceções); e (4) na presença de outras condições médicas ou de saúde mental, estas devem estar razoavelmente bem controladas. Para cirurgias de remoção das gónadas acresce o seguinte critério: (5) 12 meses contínuos de terapia hormonal (com exceção para pacientes com contraindicações médicas ou que são de algum modo incapazes ou não desejam tomar hormonas). Para as restantes cirurgias genitais, aos cinco critérios já enunciados acresce o seguinte: (6) 12 meses contínuos com experiência de vida de acordo com a identidade de género. 
tratando-se de uma população muito heterogénea, os/as profissionais devem estar atentos/as às especificidades de diferentes subgrupos (tais como, crianças/adolescentes, idosos/as, minorias étnicas/religiosas, pessoas marginalizadas ou institucionalizadas, pessoas com incapacidades...); espera-se que o/a terapeuta valide qualquer forma de expressão de género e que apoie o direito à autodeterminação do/a cliente; ou, ainda, que a intervenção deve, preferencialmente, ser promovida por equipas multidisciplinares. Raj (2002) não deixa de assinalar que nem todas as pessoas transexuais desejam e/ou necessitam de cuidados clínicos.

Lev (2004) propõe um modelo referente aos diferentes estágios que as pessoas transexuais atravessam, no que respeita ao processo de transição e à decisão de se sujeitar a tratamentos médicos orientados para a reatribuição sexual. Em paralelo, propõe objetivos terapêuticos adaptados às particulares de cada fase. Na primeira fase, na qual o indivíduo começa a ter consciência da sua eventual condição, o/a terapeuta deve procurar normalizar as experiências relacionadas com diferentes expressões de género. Na etapa seguinte é frequente a procura de recursos informativos e de apoio, sendo que parte do trabalho do/a clínico/a poderá ser auxiliar este processo. O terceiro estágio envolve a partilha da condição transgénero/transexual com outros/as significativos/as. Os objetivos terapêuticos, nesta fase, podem orientar-se para a integração familiar. A quarta etapa abrange a análise de diferentes possibilidades identitárias, no que respeita ao (trans)género, podendo a terapia ser um contexto privilegiado para esta exploração. A fase seguinte envolve as decisões concretas relativas ao processo de transição - nomeadamente acerca da vivência social concordante com o género identificado e dos eventuais tratamentos médicos a prosseguir. O/a terapeuta pode promover a tomada de decisões informadas e conscientes, apoiando as decisões do/a cliente. Por fim, tende a proceder-se à integração e à síntese da identidade, e os objetivos terapêuticos podem visar a adaptação e o apoio pós-transição.

\section{Estudo empírico}

Procurando contrariar o vazio para o qual as pessoas transexuais têm sido remetidas nas ciências sociais em Portugal (Saleiro, 2009), o presente trabalho assenta em dois propósitos: uma revisão da literatura relativa à situação clínica e social das pessoas transexuais e a apresentação de um estudo de natureza empírica. Tendo em conta a revisão da literatura apresentada, em paralelo com a escassez de dados referentes às experiências de pessoas transexuais nos serviços de saúde em Portugal, o objetivo geral do estudo empírico prende-se com a exploração das perceções de profissionais de 
saúde e de pessoas transexuais, sobre os cuidados de saúde especificamente dirigidos a pessoas transexuais em Portugal. As questões de investigação formuladas foram as seguintes:

1. Quais os perfis das pessoas que recorrem aos serviços de saúde especializados no acompanhamento de pessoas transexuais? Que profissionais trabalham nestes serviços - qual a sua formação e experiência na área?

2. De que modo é garantido o respeito pela diversidade de identidades e expressões de género na prestação de cuidados de saúde a pessoas transexuais?

3. De que forma se processam os tratamentos dirigidos à disforia de género?

3.1. Em particular, que tipo de intervenção é feito com crianças e adolescentes?

3.2 De que modo se processam as avaliações clínicas e de que forma são exercidas as funções de gatekeeping?

$\mathrm{O}$ número reduzido de serviços de saúde especializados no acompanhamento a pessoas transexuais em Portugal, à data deste estudo, conjugado com a escassez de investigações na área, conduziram-nos à opção por metodologias qualitativas. Dada a especificidade das informações que pretendemos recolher, optámos pela realização de entrevistas aprofundadas e semiestruturadas a profissionais de saúde especialistas no acompanhamento de pessoas transexuais - sendo que a maioria do corpus de análise derivou destas entrevistas Antecipando as vantagens de uma perspetiva multi-informante, foi ainda efetuado um grupo focal com pessoas transexuais.

\section{Método}

\section{Participantes}

Foram entrevistados/as seis profissionais, das áreas de psicologia clínica, psiquiatria, medicina interna e cirurgia plástica; quatro eram mulheres e dois homens, sendo que nenhum se apresentou como transexual. Por seu lado, quatro homens transexuais e três mulheres transexuais, com uma média de idades de 36 anos (variando entre os 18 e os 65 anos) participaram no grupo focal. Todos/as tinham, pelo menos, o $9^{\circ}$ ano de escolaridade, tendo um concluído o $12^{\circ}$ ano e outro uma licenciatura. Destes/as participantes três estavam empregados/as, dois estavam desempregados/as e dois estudavam. Todos/as tinham nacionalidade Portuguesa e todos/as, com exceção de uma participante, se identificaram como heterossexuais. 


\section{Técnicas de Recolha de Dados}

A escolha de técnicas de recolha de dados diferenciadas (entrevistas individuais para profissionais de saúde e grupo focal para pessoas transexuais) prendeu-se com: (1) a distinta natureza dos dados a obter junto dos dois grupos de informantes (isto é, a tecnicidade e profundidade das informações a recolher junto dos/as profissionais compeliu o recurso a entrevistas individuais); (2) a previsão das dificuldades de acesso à população transexual, que conduziu à presunção das vantagens de limitar a recolha de dados a um único momento.

A diferente natureza, tecnicidade e grau de profundidade dos dados a recolher junto dos dois grupos refletiu-se na construção dos respetivos guiões. O guião semiestruturado da entrevista aos profissionais foi desenvolvido procurando conciliar a necessidade de recolher um alargado leque de informações à essencial flexibilidade e possibilidade de adaptação a cada profissional/serviço em causa. Três dimensões foram abordadas: (i) percurso profissional do/a clínico/a, nomeadamente a sua formação, experiência de trabalho com pessoas transexuais, número e tipo de casos que acompanha ou acompanhou; (ii) dados respeitantes ao serviço, incluindo modos de funcionamento, tipos de intervenção, acolhimento dos/as utentes transexuais; e (iii) perceções sobre bem-estar e saúde mental de pessoas transexuais, diagnóstico (nomeadamente, critérios de diagnóstico e diagnóstico diferencial), processos de transição e, ainda, enquadramento legal. Por sua vez, o guião utilizado no grupo focal incluiu os seguintes tópicos: cuidados médicos e psicológicos relacionados com a condição de transexual, providos ao/à próprio/a e a familiares, desde a infância/adolescência até ao momento presente; perceção geral acerca dos cuidados de saúde prestados a pessoas transexuais; pormenorização das experiências, em contexto clínico, percebidas como positivas e negativas.

Foram contactados/as os/as profissionais de saúde que trabalhavam (ou tinham trabalhado até recentemente) nos serviços públicos especializados no acompanhamento de pessoas transexuais, num total de 8. Destes, 6 acederam a participar no estudo (taxa de resposta de 75\%). As entrevistas individuais foram realizadas nos serviços de saúde onde os/as profissionais exerciam a sua prática clínica, pela investigadora sénior, entre dezembro de 2008 e o primeiro trimestre de 2009 , com duração aproximada de 2 horas. O grupo focal com pessoas transexuais - recrutadas por um processo de bola de neve - decorreu no Centro LGBT (Lésbico, Gay, Bissexual e Transgénero), em Lisboa, em maio de 2009, e foi conduzido pelo investigador júnior e por um comoderador externo. A condução do grupo focal exigiu um esforço, por parte dos moderadores, para focar a discussão nos temas apresentados, uma vez que os/as participantes tenderam a dispersar o seu discurso para 
outras questões. Não obstante, esta dinâmica condicionou a recolha de dados junto dos/as participantes transexuais.

\section{Técnicas de Análise de Dados}

O corpus de análise, constituído maioritariamente pelas transcrições das entrevistas individuais mas também do grupo focal, foi sujeito a uma análise temática. Esta técnica de análise, ao situar-se numa posição intermédia entre a simples análise de conteúdo e a análise discursiva, apresenta algumas vantagens em relação à primeira, em especial uma maior atenção dada ao significado do discurso dos/as participantes e ao papel da linguagem (Parker, 2005). A análise temática, ao realçar os significados atribuídos pelos/as participantes às palavras e frases empregadas, emerge em formatos de apresentação dos resultados diferentes dos do derivam de análises de conteúdo (Parker, 2005). A análise decorreu em duas fases: (1) a divisão do texto em unidades de análise, derivada de forma mista - isto é, por um lado, aberta à informação que surgiu a partir dos próprios dados e, por outro, dirigida pelos respetivos guiões; (2) determinação do significado destas unidades no que respeita aos objetivos do estudo. Os resultados serão apresentados de modo a refletirem os principais temas expressos pelos/as participantes, de modo concordante com as questões de investigação referidas, bem como os significados atribuídos aos mesmos.

\section{Critérios de Rigor no Processos de Recolha e Análise}

No intuito de assegurar a qualidade dos dados recolhidos e dos resultados obtidos (em particular a sua credibilidade) foram tidos em consideração alguns dos procedimentos apontados por Lincoln e Guba (1985) para a generalidade dos métodos qualitativos: (a) triangulação de fontes, conseguida pela recolha de dados junto de profissionais de saúde especialistas bem como das próprias pessoas transexuais; (b) triangulação de investigadores/as, sendo que tanto a recolha como a análise dos dados foram realizadas por dois investigadores; (c) consulta de pares, patente em discussões de equipa com outros/as investigadores/as não diretamente envolvidos/as no estudo; (d) adequação dos referenciais, sendo que todas as entrevistas individuais bem como o grupo focal foram integralmente gravadas em aúdio, após consentimento informado dos/as participantes, e posteriormente transcritas por uma assistente de investigação; (e) consulta com a população participante, realizada de modo informal junto de membros de grupos LGBT que integraram o projeto alargado onde se insere este estudo. 


\section{Resultados}

\section{Entrevistas a profissionais de saúde}

A maioria dos/as profissionais indicou já ter acompanhado clinicamente pelo menos meia centena de pessoas transexuais. Referiram uma maior prevalência de homens transexuais nos serviços, tendência que recentemente se aproxima da igualdade numérica entre homens e mulheres. Apesar da maioria dos/as utentes estar na faixa etária dos 20-30 anos, os/as participantes acompanharam pessoas desde os 8 até cerca dos 60 anos. Entre estes/as utentes encontram-se pessoas em diversas situações no que respeita à conjugalidade, à parentalidade e à orientação sexual, isto é, pessoas solteiras ou casadas, com ou sem filhos, hetero- ou homossexuais. De acordo com as palavras de um participante, «não há qualquer divulgação de onde é que as pessoas podem acorrer se tiverem dúvidas sobre a sua identidade sexual» [E1], nomeadamente dentro da própria comunidade médica.

Todos/as os/as profissionais indicaram que se dirigem aos/às utentes transexuais de modo concordante com o género identificado e usando o nome escolhido por estes/as. Referiram, igualmente, que procuram que tal aconteça no serviço, não sendo claro se os/as próprios/as promovem iniciativas nesse sentido (por exemplo, ações de sensibilização) e não se alargando esta iniciativa para outros serviços dos mesmos hospitais. Em alguns casos, foi referido que os processos clínicos dos/as utentes transexuais são arquivados de modo separado, procurando garantir a sua confidencialidade.

Foi reportado que a intervenção feita com crianças e adolescentes pauta-se, em geral, pela atitude "esperar para ver", tal como indicado por uma participante: «[Procura-se] explicar que qualquer intervenção tem que ser adiada para uma idade em que seja possivel uma tomada de decisão mais responsável e mais amadurecida, e não influenciar, deixar as coisas acontecerem. E, sobretudo, tranquilizar a familia e tranquilizar a própria pessoa» [E5]. No entanto, não foram explicitadas as formas de apoio e suporte fornecido, nestes casos, às famílias. Os/as clínicos/as referiram que a terapêutica hormonal não é iniciada antes dos 18 anos de idade. Contudo, pelo menos dois profissionais referiram iniciar a hormonoterapia a partir dos 16 anos, desde que com consentimento do/a próprio/a e dos/as respetivos/as representantes legais: «Já tivemos casos especiais com a autorização dos pais. Contudo, a maior parte dos pais resguardam-se um bocado atrás desta lei, quase para confirmar se aquilo não é uma fase que o filho está a passar» [E3].

Uma vez no cuidado de profissionais e/ou equipas especialistas, o acesso a terapêuticas especificamente dirigidas a pessoas transexuais é condicionado por um processo de avaliação diagnóstica de Perturbação de Identidade de Género. Foi indicado por um participante: «Nunca avançamos 
para qualquer abordagem terapêutica sem dois diagnósticos feitos. Um que era feito, no nosso caso, no hospital [...] e outro de uma entidade independente» [E1]. Enquanto alguns/algumas participantes indicaram explicitamente que exigem dois diagnósticos independentes antes mesmo da terapia hormonal, uma avaliação independente foi sempre exigida antes do início da terapêutica cirúrgica. Na generalidade, o discurso dos/as clínicos/as apontou para um cuidado elevado no estabelecimento de diagnósticos claros e, consequentemente, para os efeitos a nível da morosidade do processo e das próprias desistências («(...) sendo o diagnóstico clínico, temos de ser muito prudentes, razão pela qual muitos deles achavam que nós éramos muito chatos pela forma como todos os passos do diagnóstico eram conduzidos, $e$ às vezes eram longos, muitas avaliações» [E1]; «(...) são poucos os casos que não se confirma o diagnóstico, em que nós vedamos a passagem a uma fase seguinte. [Mas] depois há aqueles que talvez não consigam chegar ao fim porque isto é um processo complicado e moroso» [E5]).

Os/as participantes relataram que os períodos de tempo necessários para proceder ao diagnóstico inicial são variáveis, desde 1 até 6 meses. Contudo, houve indicação de utentes sem diagnóstico claro mesmo ao fim de 4 anos. Estas situações foram, em geral, imputadas à dificuldade em estabelecer um diagnóstico diferencial preciso (quadros psicóticos e o fetichismo travestido excluem, para a maioria dos/as entrevistados/as, a possibilidade de determinar um diagnóstico de Perturbação de Identidade de Género). Os seguintes segmentos discursivos são exemplificativos desta situação: «O diagnóstico diferencial é feito [...] com a esquizofrenia. Componentes psicóticas em que a pessoa não é um transexual e em que acredita ser um transexual» [E3], e ainda, «Há dois casos que nós estamos convencidos, eu particularmente estou convencido, que são fetichismos travestidos e não são dois transexuais, e portanto continuam no congelador» [E1]. No que respeita à comorbidade, foi frequente o relato de situações de depressão, ansiedade social e generalizada, perturbações da personalidade, ou ideação e tentativa de suicídio. Estes problemas de saúde mental foram atribuídos ao estigma e à discriminação que as pessoas transexuais são alvo: «(...) estas pessoas fizeram um trajeto de vida habitualmente complicado, com dificuldades de integração, às vezes com coisas muito violentas, muito duras [...]. Tudo isto leva à sintomatologia ansiosa, depressiva, a determinados traços de personalidade (...)» [E5]. Alguns profissionais referiram que a própria morosidade dos processos clínicos pode ser uma agravante dos quadros depressivos e ansiosos. Por norma, para estes/as profissionais, estas sintomatologias não constituem critérios de exclusão para o diagnóstico de Perturbação de Identidade de Género. Contudo, o discurso de uma entrevistada não é claro a este respeito: «(...) podemos vir a excluir, dependendo da própria situação, da própria personalidade borderline. Até que ponto aquela personalidade está 
a interferir e é fator de exclusão para seguir com este processo. Mas isto, pronto, é discutido na própria reunião e decidimos se de facto é mais adequado ou não fazer essa exclusão» [E2].

Para a maioria dos/as clínicos/as, critérios como o estado civil, a parentalidade, a orientação sexual ou o desejo de realizar cirurgias genitais não foram referidos como relevantes para o diagnóstico. No entanto, em alguns casos, o seu relato remeteu para inconsistências entre este pressuposto e a prática que desempenham. Por exemplo, no que respeita à parentalidade, uma participante relatou: «Em relação aos filhos levantam-se algumas questões, porque efetivamente a mãe deixou de ser mãe, passou a ser um homem, portanto a figura da mãe desapareceu. (...) No fundo, a criança tem direito a ter uma progenitora» [E5]. A inexistência do desejo de realizar cirurgias genitais, mesmo que não apontado claramente por nenhum/a dos/as profissionais como critério de exclusão, não deixou de ser referido como um fator que pesa na avaliação: «Teoricamente isto não deveria influenciar negativamente o diagnóstico, mas se isso vai acontecer? Vai, porque sou humano. Porque todos quiseram fazer [cirurgias genitais] e eu teria alguma dificuldade de aceitar à priori, sem as mesmas dúvidas, que estava perante uma Perturbação de Identidade de Género» [E1].

Segundo os/as participantes, alguns/algumas dos/as utentes destes serviços adaptam as suas narrativas e histórias de vida de modo a irem de encontro ao que acreditam ser as expectativas dos/as profissionais e conseguirem, deste modo, aceder às terapêuticas: «(...) há muitos que chegam com a história bem contada, que aprenderam com outros, na internet» [E1], ou ainda «Existem transexuais que não querem cirurgia, apesar de eles acharem que têm que dizer que sim, senão em Portugal não fazem, não avançam com o processo. Um dia esperemos que consigamos evoluir também esse nivel» [E3].

O tempo que decorre entre o início dos cuidados clínicos de saúde mental até à fase das cirurgias foi igualmente descrito como muito variável. Nos casos apontados como positivos, geralmente este período rondou os 2 anos. Contudo, em alguns casos os clínicos referiram situações de mais de 4 anos.

Após este período de acompanhamento e avaliação psicológica, durante o qual se inicia a terapêutica hormonal e a experiência real de vida, é necessário apresentar um relatório à Ordem dos Médicos. Em Portugal, esta instituição faz depender da sua aprovação a realização das cirurgias de reatribuição sexual. Esta situação, apontada pelo menos por um participante como única na Europa, pode aumentar o tempo de espera para mais um ano, em alguns casos, para quem pretende realizar os tratamentos cirúrgicos. Alguns/algumas dos/as entrevistados/as manifestaram-se claramente contra esta regulamentação, alertando para o impacto que pode ter nos próprios processos clínicos: «(...) houve alguns obstáculos relativamente à Ordem, 
houve pedidos que demoraram muito tempo a seguir (...). Foi um travão desnecessário e muito prejudicial a nível clínico, porque a pessoa está pronta. Em vez de estarmos a ajudar a pessoa... Portanto, esta pessoa fez tudo, cumpriu... [E] estamos a pedir que aguarde para ter aquilo que mais deseja» [E3].

À data de realização do estudo, apenas um cirurgião plástico realizava em Portugal, no Sistema Nacional de Saúde (SNS) as ditas cirurgias de reatribuição sexual. Foram-nos relatados casos de pessoas que decidem realizar as cirurgias no estrangeiro, com elevados custos financeiros, de forma a escapar ao tempo de espera decorrente da necessidade de autorização da Ordem dos Médicos e das consequentes listas de espera para atendimento no SNS.

A maioria dos/as clínicos/as entrevistados/as referiu não ter estudado na sua formação de base matérias relacionadas com a transexualidade, e ter tido algum tipo de formação pós-graduada em sexologia, por vezes no estrangeiro. Pelo menos uma entrevistada prosseguiu estudos pós-graduados especificamente na temática da transexualidade, enquanto outros/as - apesar da formação em sexologia - referiram-se como autodidatas no que refere aos conhecimentos e competências para o trabalho com transexuais. Pelo menos três participantes realçaram que respeitam os SOC. Contudo, é de assinalar que, pelo menos em duas ocasiões, foram usadas as expressões "transexual puro" e "transexual primário", o que é contrário aos mesmos.

\section{Grupo de discussão com pessoas transexuais}

O testemunho dos/as participantes no grupo de discussão revelou a discriminação e vitimização a que as pessoas transexuais estão sujeitas nos mais variados contextos sociais, incluindo a família, e também os riscos que tal situação coloca ao seu bem-estar psicológico. Todos/as os/as participantes, à exceção de uma, estavam a avançar com o processo de transição clínica, ou já o tinham terminado, ao abrigo do SNS. De facto, uma entrevistada referiu ter procurado cuidados clínicos no estrangeiro: «Foi precisamente por causa de toda esta negatividade. Eu estive alguns meses, mas tive de desistir, porque achei que eram todos malucos. Depois mais tarde fui a Marrocos» [GF3].

Alguns/algumas participantes descreveram o apoio clínico como uma experiência claramente positiva, centrando-se especialmente na prestação dos/as clínicos/as. Os seguintes segmentos discursivos são exemplificativos: «Eu não senti nenhum comportamento de discriminação por parte dos médicos»; «No meu processo nunca fui testado, a minha médica não me tratou mal, nunca saí de lá a chorar» [GF1]; «Eu sinto-me satisfeito. O médico excelente, o psicólogo também. Logo na primeira consulta riscou o nome, 
disse que tinha que meter o nome correto (...)» [GF4] ou ainda «Fui seguido por esse médico e não podia correr melhor» [GF5].

Contudo, foram várias as situações assinaladas por diversos/as participantes como menos positivas. Por um lado, destacaram a morosidade subjacente a alguns processos: «Tive algum azar com os profissionais de saúde que tive. (...) A consulta era apenas de 6 em 6 meses e muitas vezes faltavam à consulta» [GF2]; «O que demorou mais foi o processo da Ordem dos Médicos, [só isso] demorou 7 meses» [GF4]. Por outro, apontaram para o que entendem ser prolongados processos de avaliação: «Muitas vezes estive no Instituto de Medicina Legal das $8 \mathrm{~h}$ até às $16 \mathrm{~h}$, sem comer. De manhã eram os exames psicológicos e à tarde os exames sexuais. Demorou algum tempo» [GF6]; «Eu senti-me como um ratinho de laboratório, sempre a ser testado» [GF2]. Por fim, foi também dado destaque à prestação dos profissionais de saúde, por vezes percebida como menos competente: «Eu não concordo com a terminologia que o psiquiatra que nos acompanha usa. Ele diz homem transexual para as mulheres e mulher transexual para os homens» [GF2]; "Perguntou-me que tipo de amigos é que eu tinha. Eu dava-me apenas com pessoas bissexuais e homossexuais. E a médica dizia-me que eu tinha que arranjar amigos heterossexuais, porque não me podia tornar um homem se não lidava com eles» [GF2].

\section{Discussão}

O presente estudo procurou analisar a prestação de cuidados clínicos, em Portugal, por serviços e profissionais de saúde especializados na intervenção com pessoas transexuais. Tem sido proposto (ver Israel, 2005; Raj, 2002; Sanchez et al., 2009) que os/as profissionais de saúde, nomeadamente aqueles/as que trabalham na área da saúde mental, demonstrem competência adequada no que respeita à especificidade das intervenções e consciência das vivências das pessoas transexuais. Os/as profissionais que participaram neste estudo evidenciaram, de um modo geral, ter conhecimento das sérias dificuldades que as pessoas cuja identidade de género não é concordante com o sexo atribuído à nascença atravessam (por ex., Lombardi et al., 2001), bem como do impacto que estas contrariedades têm na sua saúde mental (por ex., Nuttbrock et al., 2010). Nem todos/as os/as clínicos/as, porém, revelaram o conhecimento das orientações clínicas internacionais (WPATH, 2011) e o seguimento das mesmas: em alguns casos os cuidados de saúde estão condicionados por um número de avaliações clínicas superior ao exigido pelas recomendações da WPATH (por exemplo, duas avaliações para o início das terapias hormonais); ou ainda, quadros psicóticos ou perturbações da personalidade excluem, para alguns/algumas participantes, o início de tratamentos 
dirigidos à disforia de género - o que também é contrário aos SOC. Alguns/algumas destes/as profissionais efetuaram formações pós-graduadas com foco na temática da transexualidade no estrangeiro. Contudo, houve participantes que referiram que a sua preparação para o trabalho com transexuais advém, por um lado, da aprendizagem com colegas e, por outro, da própria prática clínica com estas pessoas. Não fica claro se, por vezes, e tal como está descrito na literatura (Korell \& Lorah, 2005), os/as utentes transexuais ao cuidado destes/as profissionais de saúde terão necessidade de os/as informar e educar no que refere à especificidade desta temática.

A prestação de cuidados clínicos sensíveis à diversidade individual e cultural está também associada à consciência do/a profissional, no que se refere ao modo como os seus próprios valores e atitudes interferem na prática clínica. Raj (2002), entre outros autores, sustentam que os/as clínicos/as que trabalham com pessoas transexuais devem fomentar a sua autoconsciência acerca do modo como os seus próprios valores se relacionam com as questões transgénero, e das suas atitudes perante a possível diversidade de expressões de género. Por vezes, nas entrevistas que efetuámos foi defendido pelos/as próprios/as profissionais que a formação e a preparação nestas matérias não pode, de facto, descurar a dimensão das atitudes. Contudo, os testemunhos dos/as participantes transexuais foram, por vezes, reveladores de conceções rígidas e estereotipadas acerca dos papéis de género, por parte dos/as profissionais que os/as acompanharam, bem como do modo como estes valores condicionam a prestação de cuidados de saúde sensíveis à diversidade. Desta forma, não é efetivamente conseguida a provisão de uma atmosfera percebida como empática e segura, livre de pressupostos (hetero)sexistas e possibilitadora da emergência de narrativas diversas. A gestão e distinção adequada dos diferentes papéis desencadeados pelos distintos processos de avaliação psicológica e psicoterapia também parecem menos bem conseguidas, sendo confusos os papéis de gatekeeping e de prestador de cuidados.

De realçar que uma das barreiras no acesso aos cuidados de saúde por parte das pessoas transexuais em Portugal, sublinhada quer pelos/as profissionais quer pelas próprias pessoas transexuais, é a necessidade de aprovação por parte da Ordem dos Médicos para a realização de cirurgias genitais.

Os resultados do presente estudo, tal como apresentados, apontam em duas direções. Por um lado, são demonstrativos de certas competências destas equipas que prestam cuidados de saúde a pessoas transexuais em Portugal. São exemplos a familiaridade com os SOC, intervenção com crianças e adolescentes, a procura de formação especializada, alguns cuidados com a confidencialidade e uso de linguagem adequada. Por outro lado, porém, os resultados também alertam para a existência de algumas práticas que são contrárias aos SOC, nomeadamente no que toca a ambiguidades na avaliação 
e diagnóstico diferencial; a interferência dos valores pessoais, nomeadamente no que respeita ao género e à sexualidade; excessivo gatekeeping; e diminuta intervenção de formação, educação e promoção da qualidade vida, independentemente das intervenções para reatribuição de sexo.

Os resultados do estudo devem ser lidos tendo em conta os limites decorrentes do seu intuito exploratório, da dimensão da amostra e das escolhas metodológicas. Em particular, a escolha do grupo focal como técnica de recolha de dados junto de pessoas transexuais não se revelou, possivelmente, a mais adequada. Futuros estudos devem ter tal facto em consideração, e optar por metodologias diferenciadas e amostras mais alargadas. Não obstante, importa salientar: a triangulação dos dados, conseguida pela recolha de informação junto de profissionais de saúde mas também das próprias pessoas transexuais; bem como a taxa de resposta de $75 \%$ no que respeita às entrevistas - apesar do número limitado de profissionais de saúde especialistas no domínio da transexualidade em Portugal. Dada a escassez de estudos referentes à situação social e clínica das pessoas transexuais no nosso país, o presente estudo poderá contribuir de modo significativo para esta área de investigação/intervenção.

\section{Referências}

Alburquerque, A. (2006). Minorias eróticas e agressores sexuais. Lisboa: Dom Quixote.

American Psychiatric Association (1994). Diagnostic and statistical manual of mental health disorders (4th ed). Washington DC: Author.

American Psychological Association. (2008). Report on the Task Force Report on Gender Identity and Gender Variance. Retirado de http://www.apa.org/ pi/lgbt/resources/policy/gender-identity-report.pdf, a 17 de Junho de 2009.

Bakker, A., van Kesteren, P .J. M., Gooren, L. J. G., \& Bezemer, P. D. (1993). The prevalence of transsexualism in the Netherlands. Acta Psychiatr Scand, 87, 237-238.

Bess, J. A., \& Stabb, S. D. (2009). The Experiences of Transgendered Persons in Psychotherapy: Voices and Recommendations. Journal of Mental Health Counseling, 31(3), 265-282.

Bockting, W., Robinson, B., Benner, A., \& Scheltema, K. (2004). Patient Satisfaction with Transgender Health Services. Journal of Sex \& Marital Therapy, 30, 277-294.

Budge, S. L., Tebbe, E. N., \& Howard, K. A. (2010). The Work Experiences of Transgender Individuals: Negotiating the Transition and Career Decision-Making Processes. Journal of Counseling Psychology, 57(4), 377-393.

Clements, K., Wilkinson, W., Kitano, K., \& Marx, R. (1999). HIV Prevention and Health Service Needs of Transgender Community in San Francisco. The Inter- 
national Journal of Transgenderism, 3(1,2). Retirado de http://www. symposion.com/ijt/hiv_risk/clements.htm a 6 de Junho de 2009.

Costa, G. C., Pereira, M., Oliveira, J. M., \& Nogueira, C. (2010). Imagens sociais das pessoas LGBT. In C. Nogueira e J. M. Oliveira (Orgs.), Estudo sobre a discriminação em função da orientação sexual e da identidade de género. (pp. 93-147). Lisboa: Comissão para a Cidadania e Igualdade de Género.

Dean, L., Meyer, I. H., Robinson, K., Sell, R. L., Sember, R., Silenzio, V. M. B., et al (2000). Lesbian, gay, bissexual and transgender health: Findings and concerns. Journal of the Gay \& Lesbian Medical Association, 4, 102-151.

Emerson, S., \& Rosenfeld, C. (1996). Stages of adjustment in family members of transgender individuals. Journal of Family Psychotherapy, 7(3), 1-12.

Ettner, R. (2007). Transsexual Couples: A Qualitative Evaluation of Atypical Partner Preferences. International Journal of Transgenderism, 10(2), 109-116.

Garrels, L., Kockott, G., Michael, N., Preuss, W., Renter, K., Schmidt, G., et al. (2000). Sex ratio of transsexuals in Germany: The development over three decades. Acta Psychiatrica Scandinavica, 102, 445-448.

Gómez-Gil, E., Trilla, A., Salamero, M., Godás, T., \& Valdés, M. (2009). Sociodemographic, clinical, and psychiatric characteristics of transsexuals from Spain. Archives of Sexual Behavior, 38(3), 378-92.

Hepp, U., Kraemer, B., Schynder, U., Miller, N., \& Delsignore, A. (2005). Psychiatric comorbidity in gender identity disorder. Journal of Psychosomatic Research, 58, 259-261.

Hines, S. (2007a). TransForming gender. Transgender practices of identity, intimacy and care. Bristol: The Policy Press.

Hines, S. (2007b). Transgendering care: Practices of care within transgender communities. Critical Social Policy, 27, 4, 462-486.

Israel, G. E., \& Tarver, D. E. (1997). Transgender care: Recommended guidelines, practical information \& personal accounts. Philadelphia, PA: Temple.

Israel, T. (2005). ... and sometimes T: Transgender issues in LGBT psychology. Newsletter of the Society for the Psychological Study of Lesbian, Gay, and Bisexual Issues, 21 (3), 16-18.

Johnson, K. (2007). Transsexualism: Diagnostic Dilemmas, Transgender Politics and the Future of Transgender Care. In V. Clarke \& E. Peel (Eds.). Out in Psychology. Lesbian, Gay, Bisexual, Trans and Queer Perspectives (pp. 444-464). Washington, D.C.: American Psychological Association.

Kenagy, G. P. (2005). Transgender Health: Findings from Two Needs Assessment Studies in Philadelphia. Health and Social Work, 30(1), 19-26

Knudson, G., De Cuypere, G., \& Bockting, W. (2010). Recommendations for revision of the DSM diagnosis of gender identity disorders: Consensus statement of the World Professional Association for Transgender Health. International Journal of Transgenderism, 12, 115-118.

Korell, S. C., \& Lorah, P. (2007). An Overview of Affirmative Psychotherapy and Counseling with Transgender Clients. In Bieschke, K., Perez, R. \& DeBord, K. (Eds.). Handbook of counseling and psychotherapy with lesbian, gay, and bi- 
sexual clients, 2nd Edition (pp. 271-288). Washington, D.C.: American Psychological Association.

Lawrence, A. A. (2008). Gender identity disorders in adults: Diagnosis and treatment. In D. L. Rowland \& L. Incrocci (Eds.), Handbook of sexual and gender identity disorders (pp. 423-456). New York: Wiley.

Lev, A. I. (2004). Transgender emergence: Therapeutic guidelines for working with gender-variant people and their families. New York: Haworth Clinical Practice Press.

Lev, A. I. (2007). Transgender Communities: Developing Identity through Connection. In Bieschke, K., Perez, R. \& DeBord, K. (Eds.). Handbook of counseling and psychotherapy with lesbian, gay, and bisexual clients, 2nd Edition (pp. 147-175). Washington, D.C.: American Psychological Association.

Lincoln, Y. S., \& Guba, E. G. (1985). Naturalistic Inquiry. Newbury Park, CA: Sage Publications.

Lombardi, E. L.,Wilchins, R. A., Priesing, D., \& Malouf, D. (2001). Gender violence: Transgender experiences with violence and discrimination. Journal of Homosexuality, 42(1), 89-101.

Maguen, S., \& Shipherd, J. (2010). Suicide risk among transgender individuals. Psychology \& Sexuality, 1(1), 34-43.

May, K. (2002). Becoming Women: Transgendered Identities, Psychosexual Therapy and the Challenge of Metamorphosis. Sexualities, 5(4), 449-464.

Newfield, E., Hart, S. Dibble, S., \& Kohler, L. (2006). Female-to-male transgender quality of life. Quality of Life Research, 15, 1447-1457.

Nuttbrock, L., Hwahng, S., Bockting, W., Rosenblum, A., Mason, M., Macri, M., \& Becker J. (2010). Psychiatric impact of gender-related abuse across the life course of male-to-female transgender persons. Journal of Sex Research, 47(1), 12-23.

Olsson, S. E., \& Moller, A. R. (2003). On the incidence and sex ratio of transsexualism in Sweden, 1972-2002. Archives of Sexual Behavior, 32, 381-386.

Parker, I. (2005). Qualitative Psychology: Introducing Radical Research. Maidenhead: Open University Press.

Rachlin, K. (2002). Transgender Individual's Experiences of Psychotherapy. The International Journal of Transgenderism, 6(1). Retirado de http://www.symposion.com/ijt/ijtvo06no01_03.htm a 4 de Junho de 2009.

Raj, R. (2002). Towards a transpositive therapeutic model: Developing clinical sensitivity and cultural competence in the effective support of transsexual and transgendered clients. The International Journal of Transgenderism, 6(2). Retirado de http://www.symposion.com/ijt/ijtvo06no02_04.htm a 3 de Junho de 2009.

Saleiro, S. (2009). Transexualidade e transgénero em Portugal: dois "vazios" em debate. In M. C. Silva et al (Orgs.), Actas do X Congresso Luso-Afro Brasileiro de Ciências Sociais, Sociedades Desiguais e Paradigmas em Confronto, Vol. IV - Família, Género e Sexualidades, pp. 84-90. 
Sánchez, F. J., \& Vilain, E. (2009) Collective self-esteem as a coping resource for male-to-female transsexuals. Journal of Counseling Psychology, 56(1), 202-209 .

Sanchez, N. F., Sanchez, J. P., \& Danoff, A. (2009). Health Care Utilization, Barriers to Care, and Hormone Usage Among Male-to-Female Transgender Persons in New York City. American Journal of Public Health, 99(4), 713-719.

Silverman, D. (2006). Interpreting qualitative data (3rd ed.). London: Sage.

Sue, D. W., Arredondo, P., \& McDavis, R. J. (1992). Multicultural Counseling Competencies and Standards: A Call to the Profession. Journal of Counseling \& Development, 70(4), 477-486.

Vale de Almeida, M. (2010). O contexto LGBT em Portugal. In C. Nogueira e J. M. Oliveira (Orgs.), Estudo sobre a discriminação em função da orientação sexual e da identidade de género. (pp. 45-92). Lisboa: Comissão para a Cidadania e Igualdade de Género.

Vocks, S., Stahn, C., Loenser, L., \& Tegenbauer, U. (2009). Eating and body image disturbances in male-to-female and female-to-male transsexuals. Archives of Sexual Behavior, 38(3), 364-377.

World Professional Association for Transgender Health (2011). Standards of Care for the Health of Transsexual, Transgender, and Gender Nonconforming People. $7^{\text {th }}$ Version. Retirado de http://www.wpath.org/documents/Standards \%20of\%20Care\%20V7\%20-\%202011\%20WPATH.pdf a 15 de Outubro 2011. 